\begin{tabular}{|c|c|c|c|}
\hline Eiszeitalter u. Gegenwart & 38 & $\begin{array}{c}115-127 \\
\text { 5 Abb., 1 Tab. }\end{array}$ & Hannover 1988 \\
\hline
\end{tabular}

\title{
Hinweise auf eine saalezeitliche Warmzeit am Niederrhein
}

\author{
Josef Klostermann, Hans-Wolfgang Rehagen \& Ulrike Wefels *)
}

Borehole sections, Pleistocene, lithostratigraphy, sand, gravel, silt, core,

heavy mineral analysis, pollen diagram, floral list, oak mixed forest, biostratigraphy, Saalian, deglaciation

Rhine Westphalian Basin, North Rhine-Westphalia

\begin{abstract}
Kurzfassung: Eine Kernbohrung im Bereich der nördlichen Niederrheinischen Bucht, etwa $1,5 \mathrm{~km}$ östlich der Ortschaft Weeze, erbrachte eine Schichtenfolge, die mit den Rinnenschottern der Elster-Kaltzeit beginnt und über die eemzeitlichen Torfe von Weeze bis hin zu den Kiesen und Sanden der Älteren Niederterrasse reicht. Zwischen holsteinzeitlichen Ablagerungen und eemzeitlichen Torfen wurden mehrere humose Lagen erbohrt. Der größte Teil von ihnen dürfte umgelagert sein. Eine Ausnahme bildet ein aus schluffigen Feinsanden bestehender Abschnitt, die Vorselaer-Schichten, die etwa 0,5 m unter den eemzeitlichen Torfen erbohrt wurden. Bei diesen Schichten handelt es sich um Ablagerungen, die die Pollenflora einer warmzeitlichen Vegetation mit EMW (überwiegend Quercus), Taxus, Corylus und viel Alnus sowie Hedera und Ilex enthalten und nach ihrer Position innerhalb der Schichtenfolge ans Ende der Saale-Kaltzeit zu stellen sein dürften.
\end{abstract}

[Hints to a Saalian Warm Period at the Lower Rhine Embayment]

\begin{abstract}
A borehole in the area of the Lower Rhine embayment, approximately $1,5 \mathrm{~km}$ to the east of the village Weeze, provided a succession, beginning with the channel gravel of the Elsterian up to the Eemian peats of Weeze, which are covered by gravel and sand of the older Lower Terrace. In between the succession of the Holsteinian sediments and the Eemian peats, some mouldy layers were found. Most of the mouldy layers seem to be reassorted except of the uppermost silty sands, the Vorselaer-beds, which are situated about $0,5 \mathrm{~m}$ below the Eemian peats. The Vorselaer-beds are sediments which contain a thermophilous pollenflora with mixed oak forest (most of it Quercus), Taxus, Corylus, much Alnus, some Hedera and Ilex that, according to their position within the succession, are tentatively placed at the end of the Saalian.
\end{abstract}

*) Anschrift der Autoren: Dr. J. KlostermanN, Dr. H.-W. Rehagen, U. WeFeLs, Geologisches Landesamt NW, De-Greiff-Str. 195, 4150 Krefeld.

\section{Einleitung}

Zur Erkundung der quartären Schichtenfolge des nördlichen Niederrheins wurden im Rahmen der geologischen Landesaufnahme auf den Blättern 4403 Geldern, 4404 Issum, 4304 Xanten und 4303 Uedem der TK 25/139 Spülbohrungen mit Teufen von $40 \mathrm{~m}$ bis $90 \mathrm{~m}$ niedergebracht. Von dem erbohrten Material wurden inzwischen ca. 1500 Proben schwermineralanalytisch untersucht. Erste Ergebnisse dieser Untersuchungen wurden in den Erläuterungen zur Geologischen Karte 1:25000 4403 Geldern (KLOSTERMANN 1984) und 4404 Issum (KLOSTERMANN 1986) sowie von KLOSTERMANN (1985) veröffentlicht. Pollenanalytische Untersuchungen wurden bisher nur an wenigen Stellen durchgeführt, da sie an Probenmaterial aus Spülbohrungen nicht sinnvoll sind.

Im Jahre 1985 konnten erstmals im Auftrage des Geologischen Landesamtes NW Kernbohrungen bis zu einem Teufenbereich von etwa $20 \mathrm{~m}$ durchgeführt werden. Daraufhin wurde versucht, im Blattgebiet 4303 Uedem der TK 25 in der Nähe der Ortschaft Weeze (siehe Abb. 1) das Liegende des dort nachgewiesenen Eems (VON DER BRELIE \& MÜCKENHAUSEN \& REIN 1955) mit Hilfe einer Kernbohrung zu untersuchen. Die an dieser Stelle zunächst abgeteufte Spülbohrung mit der Bezeichnung KBB 7 zeigte, daß sich im Liegenden des eemzeitlichen Torfes mehrere Schluff- und Feinsandschichten mit humosen Lagen befanden. Mit Hilfe einer wenige Meter entfernt angesetzten Kernbohrung (KB 7 B) sollte nun versucht werden, gekerntes Probenmaterial für pollen- und schwermineralanalytische Untersuchungen zu gewinnen. Die Ergebnisse zeigten, daß die humosen Lagen eine thermophile Flora enthielten. Es stellte sich nun die Frage, ob es sich bei diesen Lagen um autochthone saalezeitliche Bildungen handelte oder ob 


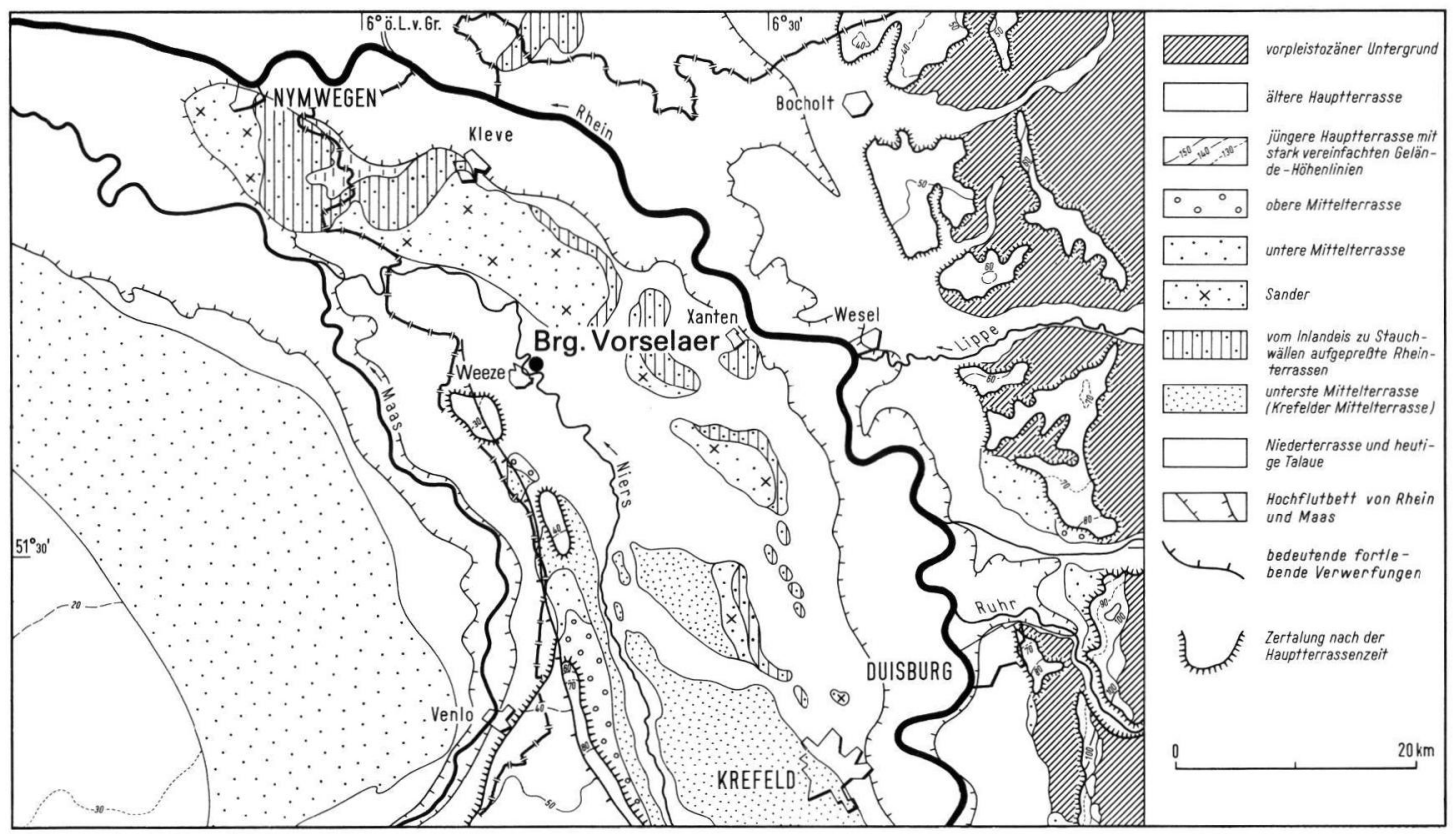

Abb. 1: Terrassengliederung im niederrheinischen Tiefland (nach BRAUN \& QUITZOW 1961).

man es mit umgelagerten Resten der HolsteinWarmzeit zu tun hatte.

Um dieses Problem zu lösen, wurde etwa $5 \mathrm{~m}$ von der ersten Kernbohrung (KB $7 \mathrm{~B}$ ) entfernt eine weitere Kernbohrung, die Bohrung KB 7 C, abgeteuft, die die vollständige quartäre Schichtenfolge bis ins Tertiär erschließen sollte. Der Kernmarsch der Bohrung KB 7C begann aus technischen Gründen erst in $4 \mathrm{~m}$ Tiefe. $\mathrm{Da}$ die Untersuchungsergebnisse der Bohrungen $\mathrm{KB} 7 \mathrm{~B}$ und $\mathrm{KB} 7 \mathrm{C}$ ausgezeichnet übereinstimmen und beide Bohrungen nur $5 \mathrm{~m}$ voneinander entfernt sind, wird im folgenden so verfahren, daß beide Bohrungen kombiniert dargestellt werden. Die oberen 5,25 $\mathrm{m}$ der Schichtenbeschreibung entstammen der Bohrung KB 7B, der Rest der Bohrung KB 7C. Die Kombination beider Bohrungen erhielt die Bezeichnung Bohrung Vorselaer $\left(\mathrm{KB}^{\mathrm{B}} / \mathrm{C}\right)$.

\section{Untersuchungsmethoden}

\subsection{Schwermineralanalyse}

Aus dem tonigen bzw. sandigen Probenmaterial der Bohrungen wurde jeweils die Fraktion

$0,036 \mathrm{~mm}$ bis $0,315 \mathrm{~mm}$ bzw. die Fraktion

$0,063 \mathrm{~mm}$ bis $0,315 \mathrm{~mm}$ durch Naßsiebung

abgetrennt.
Auf eine chemische Vorbehandlung der Proben wurde verzichtet, um das vollständige Schwermineralspektrum zu erhalten. Je nach Bedarf wurden 5 bis $20 \mathrm{~g}$ des so gewonnenen getrockneten Materials der Schweretrennung in einem Scheidetrichter mit $200 \mathrm{ml}$ Fassungsvermögen unterzogen. Als Schwereflüssigkeit diente Bromoform mit einer Dichte von $2,86 \mathrm{~g} / \mathrm{cm}^{3}$. Nach vollendeter Separation (Absetzzeit mindestens 2 Stunden) wurde die Schwerefraktion entnommen.

Zur anschließenden mikroskopischen Bestimmung der transparenten Schwerminerale wurden Dauerpräparate hergestellt. Als Einbettungsmittel diente Aroclor $\left(\mathrm{n}_{\mathrm{D}}=1,665\right)$. Je Präparat wurden entlang paralleler Linien oder mittels der Gesichtsfeldmethode ca. 200 Körner ausgezählt. Die Ergebnisse der Untersuchungen wurden in Form von Schwermineraldiagrammen, die die Analysenergebnisse in Abhängigkeit von der Teufe wiedergeben (vgl. Abb. 2), dargestellt.

\subsection{Pollenanalyse}

Aus dem inneren Bereich der Bohrkerne wurde eine $2-3 \mathrm{~cm}^{3}$ große Probenmenge entnommen und, soweit erforderlich, 24 Stunden lang mit $38 \%$ iger kalter Flußsäure behandelt. Die Aufbereitung erfolgte ansonsten nach der Acetolyse-Methode von ERDTMAN. Pro Präparat wurde in einem Wasser-Glyceringemisch bei 400 facher Vergrößerung (Ortholux) auf ca. 300 Baumpollen ausgezählt und darüber hinaus weiteres 
Tab. 1: Gliederung der Elster- und Saale-Kaltzeit

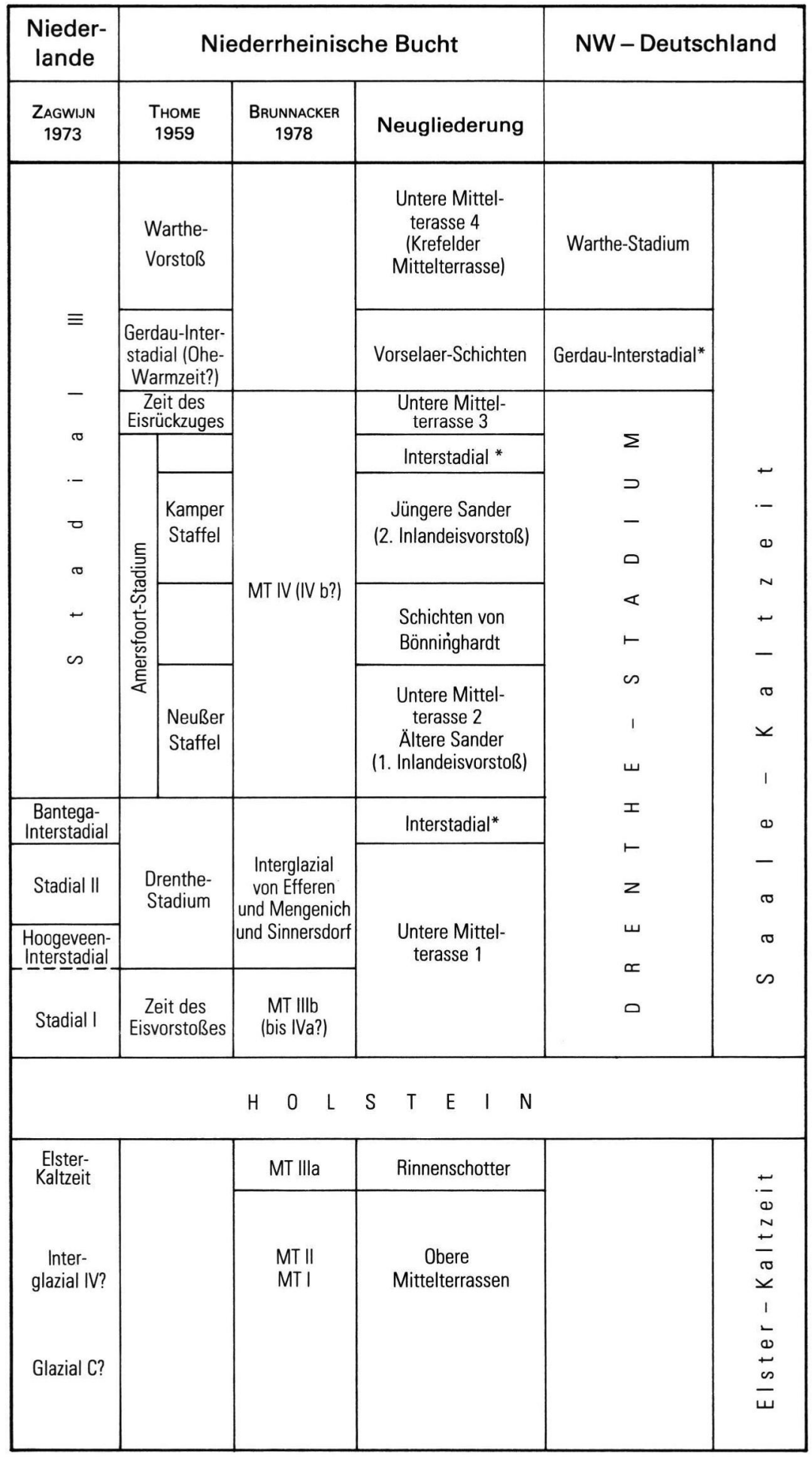


Material durchgemustert, um auch seltenere Formen $\mathrm{zu}$ erfassen. Als Berechnungsgrundlage dient die Summe aller Baum- und Nichtbaumpollen $=100 \%$; auf diese Basissumme sind die Prozentangaben für Wasser- und Sumpfpflanzen sowie für die Sporen bezogen. Die mazerierten Probenrückstände werden in Glycerin aufbewahrt.

\section{Geologischer Überblick}

Die spätelsterzeitlichen Rinnenschotter lassen sich aufgrund ihres hohen Klinopyroxengehaltes ausgezeichnet miteinander verknüpfen. So ist es auch dann möglich, die im Hangenden der Rinnenschotter anschließenden Sedimente der Holstein-Warmzeit zu identifizieren, wenn sie nicht tonig entwickelt sind, sondern deren sandig-kiesiges Äquivalent ausgebildet ist.

Im Bereich der Niedertheinischen Bucht können vier saalezeitliche Terrassenkörper und zwei Inlandeisvorstöße nachgewiesen werden (vgl. Tab. 1). Die Untere Mittelterrasse 1 ist am nördlichen Niederrhein durch spätere Erosion vollständig beseitigt. Die ältesten saalezeitlichen Ablagerungen gehören dort der Unteren Mittelterrasse 2 an (KLOSTERMANN 1986).

Während der Aufschotterung der Unteren Mittelterrasse 2 kam es zu zwei Inlandeisvorstößen in die Niederrheinische Bucht. Obwohl sich Sanderablagerungen und der Terrassenkörper der Unteren Mittelterrasse 2 schwermineralanalytisch nur wenig voneinander unterscheiden, zeichnen sich doch gewisse Tendenzen in den Schwermineralspektren ab, die eine Zuordnung zur Unteren Mittelterrasse 2 oder zum Sander wahrscheinlicher machen.

Im Bereich Vorselaer (siehe Abb. 1) wurden im Liegenden eemzeitlicher Torfe und im Hangenden von Äquivalenten der Holstein-Warmzeit mehrere Schichten mit einer warmzeitlichen Flora erbohrt, die in die Saale-Kaltzeit einzuordnen sind. Diese Schichten, die hier als „Vorselaer-Schichten” bezeichnet werden, wurden vermutlich nach den saalezeitlichen Eisvorstößen abgelagert.

\subsection{Rinnenschotter und Holstein-Warmzeit}

Die $\mathrm{R}$ i n n e n s h o t t e r bestehen vorwiegend aus grauen, feinkiesigen Mittel- und Grobsanden, die fast immer einen gewissen Feinsand-Anteil aufweisen. Der unterste Teil der Rinnenschotter ist häufig als Grobkies-Lage mit Steinen ausgebildet. Die Kiese sind infolge intensiver Verwitterung von Eisen- und Mangan-Krusten umschlossen. Außerdem tritt an der Basis häufig aufgearbeiteter Muschelschill auf.
Die Schwermineralzusammensetzung der Rinnenschotter zeichnet sich durch die höchsten Klinopyroxengehalte im Bereich des nördlichen Niederrheins aus. Sie schwanken zwischen $40 \%$ und $68 \%$ und erlauben eine sichere Korrelation dieser Schichten in verschiedenen Bohrungen (vgl. Abb. 2). Die Klinopyroxene der Rinnenschotter sind, gegenüber den in anderen quartären Schichten auftretenden, auffallend groß und außerdem stark gezahnt.

Über den Rinnenschottern schließen sich die Schichten der $\mathrm{Hols}$ te in-W a r m z e it an. In der Bohrung WW Kempen (vgl. Abb. 2) wurden graugrüne, stark karbonatische Tone und Schluffe mit eingelagerten Torfen erbohrt. Der unterste Teil der Schichtenfolge besteht dort aus grau bis graubraun gefärbten, karbonatischen, grobsandigen Fein- und Mittelsanden.

In der Bohrung Vorselaer (KB $7^{\mathrm{B} / \mathrm{C})}$ wurde offenbar nur dieser untere Teil der Schichtenfolge der Holstein-Warmzeit erbohrt. Dort dürften die unmittelbar im Hangenden der Rinnenschotter nachgewiesenen grauen bis gelbgrauen Fein- und Mittelsande, die immer einen gewissen Grobsand- und Feinkies-Anteil führen, die Äquivalente der Holstein-Warmzeit sein. Auch KEMPF (1966: 15) beschreibt im untersten Teil der „Krefelder Schichten” Grob- und Mittelsande mit wenig Fein- und Mittelkies.

Das Schwermineralspektrum des sandigen Abschnittes der Holstein-Warmzeit ist durch einen EpidotAnteil von $25 \%$ bis $35 \%$ charakterisiert. Die Werte der grünen Hornblende schwanken zwischen $30 \%$ und $40 \%$. Auffälligstes Merkmal des sandigen Holsteins ist ein deutlich höherer Klinopyroxen-Anteil als in der darüberliegenden Schichtenfolge des tonigschluffigen Holsteins. Die Proben aus dem unteren sandigen Abschnitt der Holstein-Warmzeit sind häufig stark durch Karbonat und Eisenverbindungen verunreinigt. Kennzeichnend ist außerdem ein hoher Glimmer-Anteil. Besonders deutlich ist dieser untere Abschnitt in der Bohrung WW Kempen (vgl. Abb. 2) erkennbar.

Das Pollendiagramm der Bohrung WW Kempen (siehe Abb. 3) setzt bei 17,7 m mit einer klaren PinusDominanz von $37,7 \%$ unter den Baumpollen ein. Alnus erreicht $18,0 \%$. Die Anteile des Eichenmischwaldes (EMW), allein durch Quercus vertreten, von Corylus, Picea und Betula liegen zwischen 2,0 und 6,6\%. Carpinus kommt nur mit einem Pollenkorn vor, und Abies fehlt noch. In diesem Niveau befindet sich auch der einzige Fund von Pterocarya mit 1,6\%. Die Anteile der Nichtbaumpollen sind etwas erhöht. Doch gehen sie nur auf wenige Sippen zurück.

Die Pollenspektren zwischen 16,8 und 11,4 m zeigen eine deutliche Alnus-Dominanz mit Pinus in Sub- 
Bohrung WW Kempen

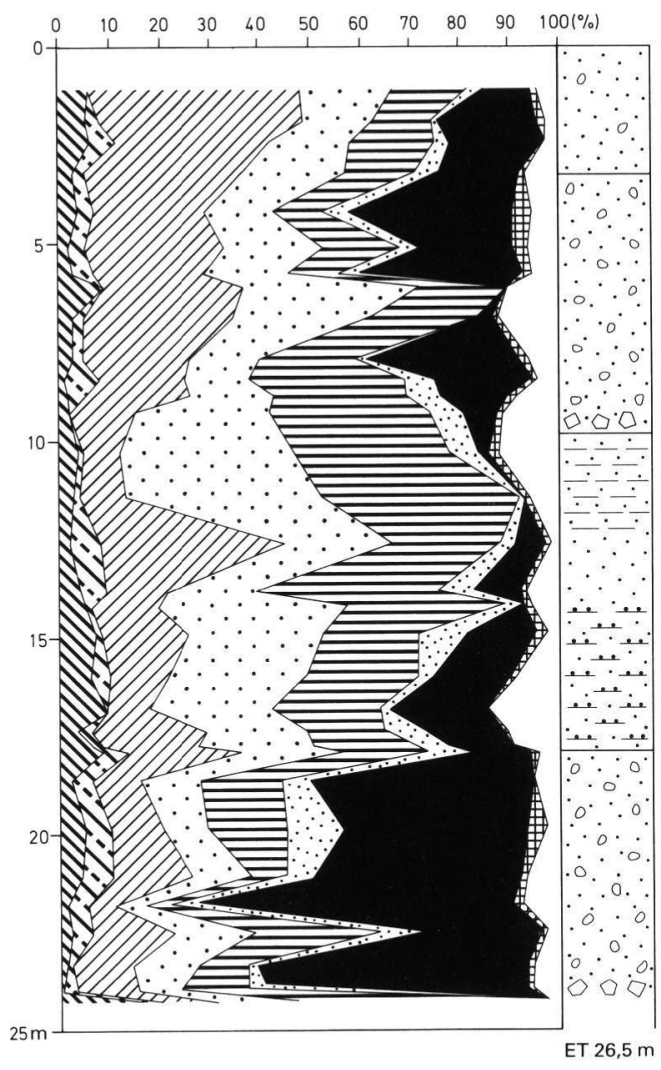

$\mathrm{Niederterrasse}$

Untere Mittelterrasse 3

Holstein-Warmzeit

Rinnenschotter

(Mittlere Mittelterrasse)

Schwerminerale

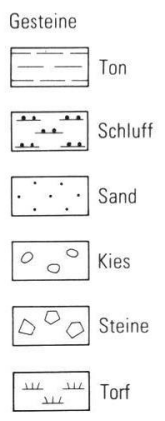

ET Endteufe

Abb. 2: Schwermineralprofil des Pleistozäns in der Bohrung Wasserwerk Kempen;

TK 25: 4504 Kerken, R 32696 H 96908.

dominanz. Die Erlenkurve steigt anfangs stetig an und gipfelt zweimal knapp über $40 \%$. Die Kiefer fällt dagegen steil unter $15 \% \mathrm{ab}$, erreicht dann im oberen Teilabschnitt wieder Werte zwischen 22,9 und $31,8 \%$.

Im EMW finden sich jetzt auch Ulmus, Tilia und Fraxinus ein. Ihre Pollenkurven sind jedoch nicht geschlossen. Nach wie vor ist Quercus allein regelmäßig vertreten. Zeitweise liegt auch Corylus über $10 \%$. Carpinus nimmt stetig zu und erreicht maximal $9,5 \%$, um dann infolge des Alnus-Gipfels und des erneuten Pinus-Anstiegs zurückgedrängt zu werden. Inzwischen hat sich auch Abies eingefunden und bildet jetzt eine geschlossene Kurve, die jedoch meist nur unter $2 \%$ verläuft. Gleichmäßig verläuft die 


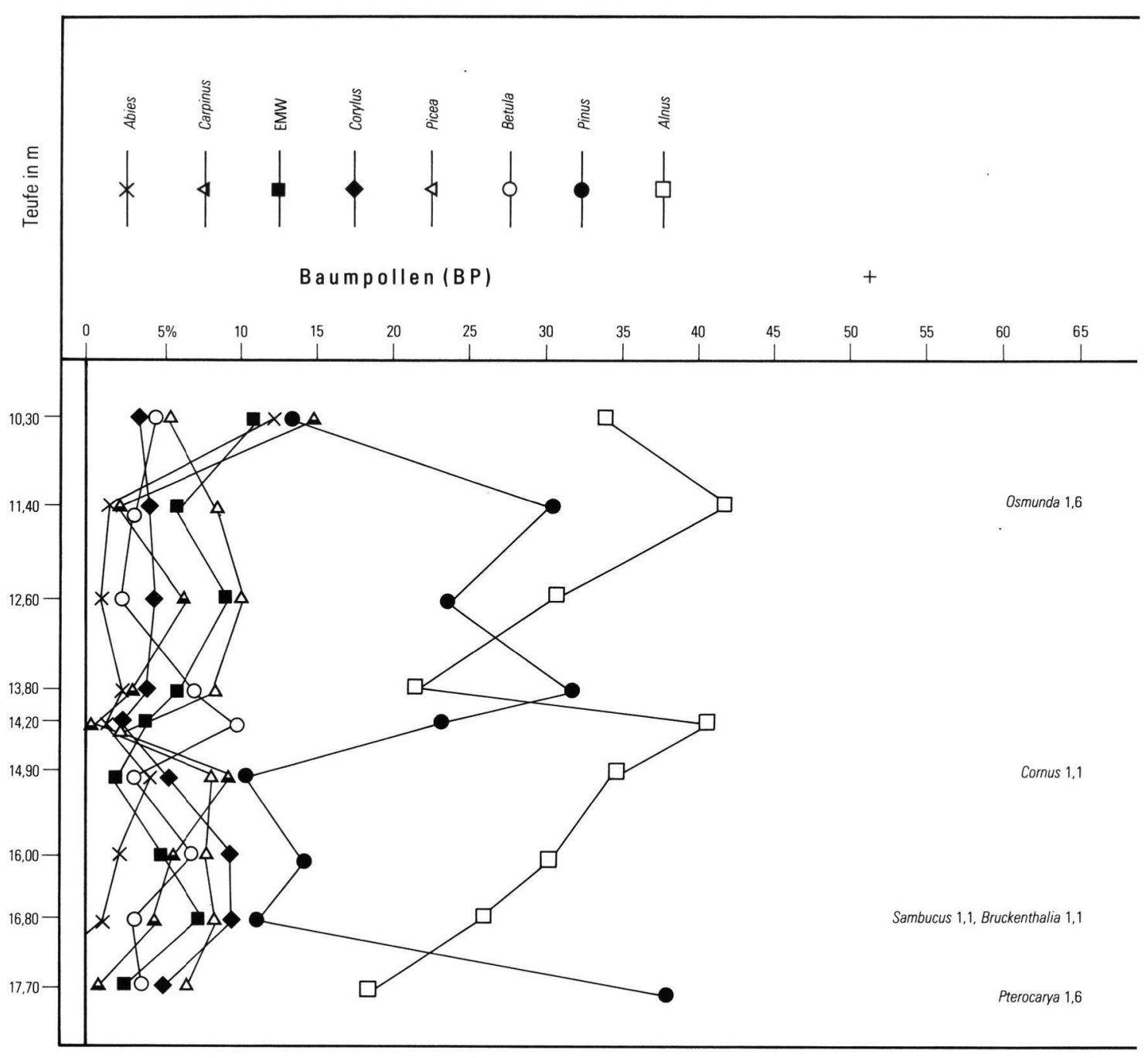

Abb. 3: Pollendiagramm der Bohrung WW Kempen (vgl. Abb. 2).

Picea-Kurve, sieht man von einer kurzen Depression bei $14,2 \mathrm{~m}$ ab, die durch kräftige Kurvenanstiege von Alnus, Pinus sowie Betula auch bei den anderen BPKurven hervorgerufen wird. Vorübergehend finden sich Salix und Frangula ein und dürften lokal ein Weiden-Faulbaumgebüsch gebildet haben. Der Anteil der NBP geht stetig zurück. Einmal wurde Bruckenthalia beobachtet. Der Anstieg der Wasserund Sumpfpflanzen geht fast ausschließlich auf die Ausbreitung der Typhaceae zurück.

Bei 10,3 m kündigen sich Veränderungen im BP-Spektrum an. Die Kurven von Carpinus und Abies steigen kräftig auf 14,4 bzw. 12,2\% an, was auf Kosten von Pinus, Alnus und Picea geschieht. Diese Entwicklung läßt sich jedoch nicht weiter verfolgen, da sich die Schichten im Hangenden als pollenleer erwiesen.
Im vorliegenden Pollendiagramm ist ein warmzeitlicher Vegetationsabschnitt erfaßt, der durch eine Alnus-Pinus-Dominanz gekennzeichnet ist sowie durch das Einwandern und Ausbreiten von Carpinus und Abies. Die parallel laufende Schwermineralanalyse weist für diese Sedimente ein holsteinzeitliches Alter aus. Vergleicht man die pollenanalytischen Untersuchungsergebnisse mit denen aus den benachbarten Niederlanden, so zeigt sich eine gute Übereinstimmung. Die erfaßte Vegetationsentwicklung gehört in die Übergangsphase von Ho 2 nach Ho 3 (ZAGWIJN 1973). Nach MÜLLER (1974) und seiner Arbeit über das Holstein-Interglazial von Munster-Breloh lassen sich die vorliegenden Ergebnisse mit der Zone IX parallelisieren und der steile Abfall der Pinus-Kurve zu Beginn des Pollendiagramms mit dem Ende der Zone VIII. 


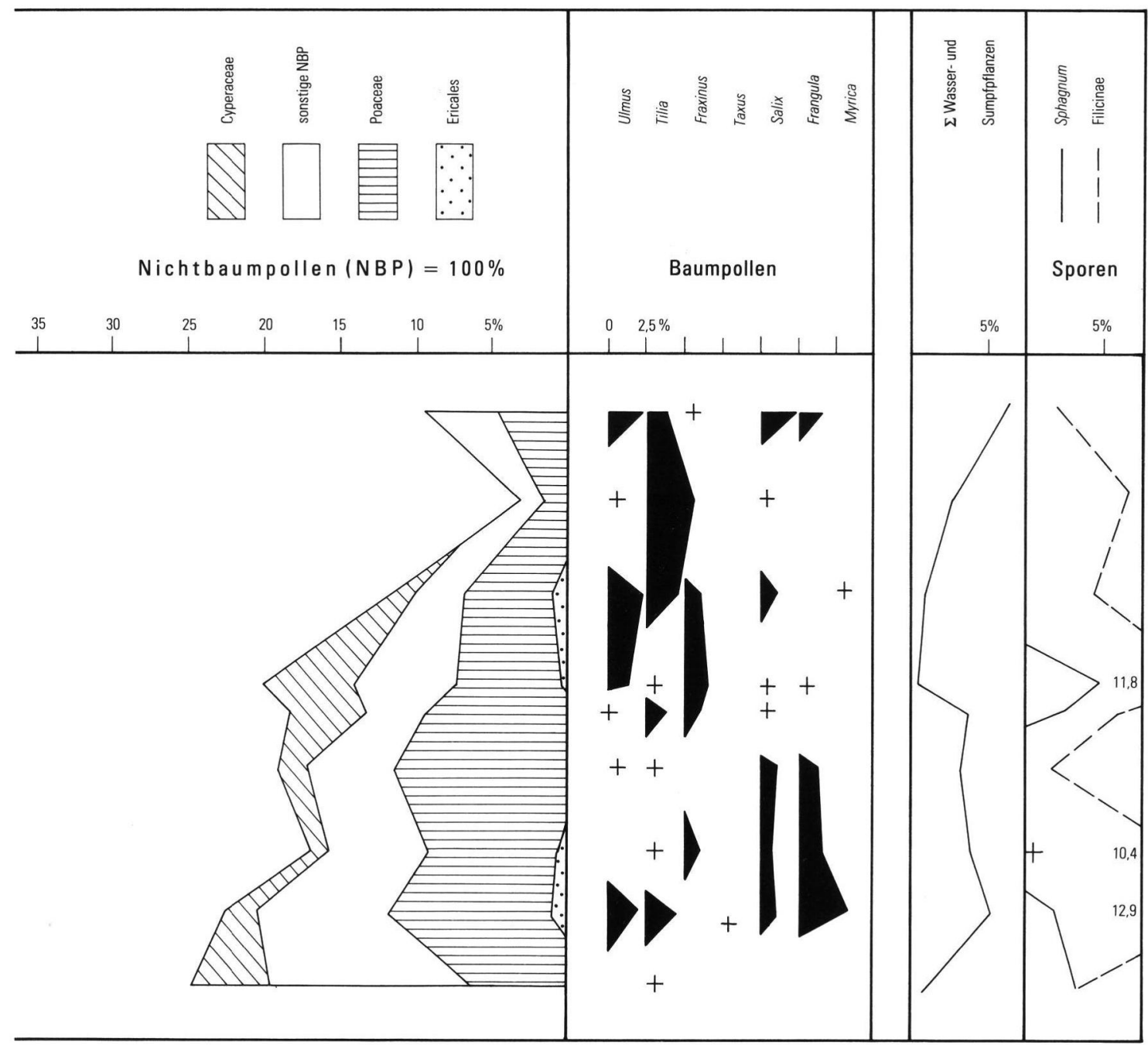

\subsection{Die Schichtenfolge der Bohrung Vorselaer} $\left(\mathrm{KB}^{\mathrm{B}} / \mathrm{C}\right)$

Die Quartär- / Tertiär-Grenze wurde in der Bohrung Vorselaer bei 20,73 m durchörtert (siehe Abb. 4). Die tertiären Schichten bestehen dort aus dunkelgrüngrauen, stark glimmerführenden Feinsanden, die keine Schichtung zeigen. Das Schwermineralspektrum dieser Schichten zeigt ein deutliches Überwiegen des stabilen Anteils und ein vollständiges Fehlen der vulkanischen Komponenten.

Von $19,32 \mathrm{~m}$ bis $20,73 \mathrm{~m}$ wurden stark von Grobkiesen durchsetzte Fein- und Mittelkiese der Rinnenschotter erbohrt, die von Mangan-Krusten umschlossen sind. Dieser Schichtenabschnitt kann mit dem untersten Teil der im Schacht Tönisberg nachgewie- senen Rinnenschotter (KEMPF 1966: 10) sowie den Rinnenschottern in der Bohrung WW Kempen verknüpft werden. Im untersten Teil der Bohrung WW Kempen bestehen die Rinnenschotter aus Grobkiesen und Steinen. Infolge Aufarbeitung tertiärer Schichten ist der Klinopyroxenanteil in den untersten Abschnitten der Bohrung Vorselaer durch den Einfluß stabiler Schwerminerale relativ zurückgedrängt. Zwischen 12,00 $\mathrm{m}$ und 19,32 $\mathrm{m}$ wurde die typische Fazies der Rinnenschotter durchbohrt. Es sind graue und braungraue, feinkiesige Mittel- und Grobsande. Im obersten Teil der Schichtenfolge zeigt der Klinopyroxen zwei deutliche Maxima, eines von $41 \%$ bei $12,85 \mathrm{~m}$ und eines von $28 \%$ bei $14,45 \mathrm{~m}$.

Zwischen 10,56 m und 12,00 m wurden gelbgraue und braungraue Mittel- und Grobsande durchbohrt. 
Bohrung Vorselaer (KB 7B/C)

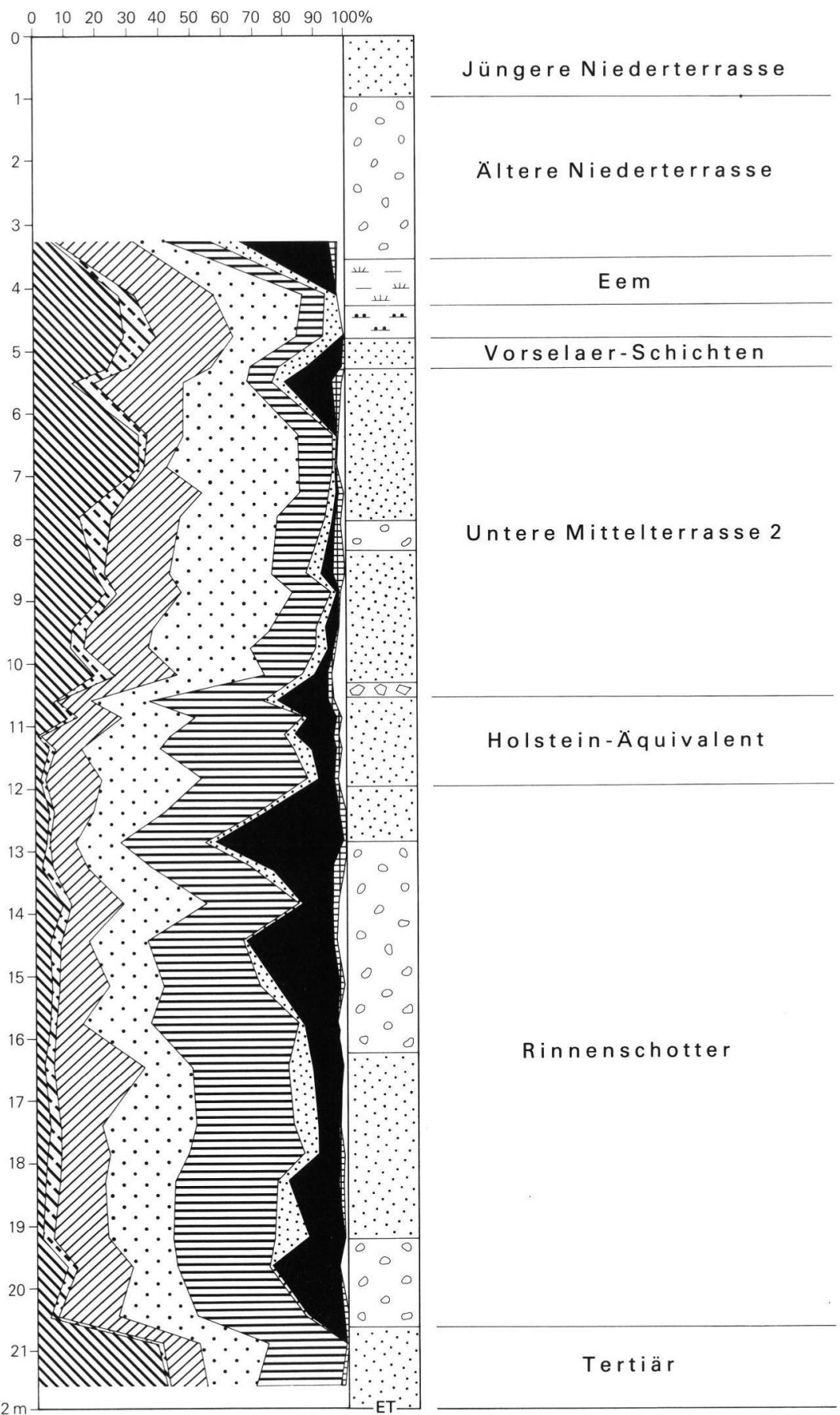

Abb. 4: Schwermineralprofil der Bohrung Vorselaer ( $\left.\mathrm{KB} 7^{\mathrm{B}} / \mathrm{C}\right)$; TK 25: 4303 Uedem, R 15 170, H 21130 (Legende s. Abb. 2). 
Im obersten Teil sind die Schichten rotbraun gefärbt. Das Schwermineralspektrum zeigt die typischen Merkmale des sandigen Abschnittes der HolsteinWarmzeit (vgl. Kap. 3.1.). Auch andere typische Eigenschaften, wie die starke Verunreinigung durch Eisenverbindungen sowie der charakteristische hohe Glimmer- und Karbonat-Gehalt, treten deutlich hervor. Diese Schichtenfolge stellt eindeutig das untere sandige Äquivalent der Holstein-Warmzeit dar.

Zwischen 5,27 m und 10,56 m wurden Schichten der Unteren Mittelterrasse 2 durchbohrt.

Direkt im Hangenden des Holstein-Äquivalents wurde zwischen 10,33 m und 10,56 m eine Steinsohle erbohrt. Diese Steinsohle konnte regelmäßig an der Basis der Unteren Mittelterrasse 2 beobachtet werden.

Darüber folgt zwischen 7,37 m und 10,33 m eine aus Mittelsanden und Feinkiesen bestehende Schichtenfolge, die mit den Schwermineralspektren der Unteren Mittelterrasse 2 zu verknüpfen ist.

Zwischen 5,93 $\mathrm{m}$ und 7,37 $\mathrm{m}$ schließen sich gelbbraun gefärbte Feinsande an, die durch rostfarbene Bändchen fein geschichtet erscheinen. Das Schwermineralspektrum dieser Feinsande zeigt einen kräftigen Anstieg der stabilen Schwerminerale (vgl. Abb. 4), der durch die Aufarbeitung von Schmelzwassersedimenten verursacht worden sein dürfte. Die Ausbildung der Schwermineral-Körner, die petrographische $\mathrm{Zu}$ sammensetzung sowie die Pollendiagramme der gesamten Schichtenfolge der Unteren Mittelterrasse 2 zeigen, daß der größte Teil dieser Schichten aus umgelagertem Sander- und Holstein-Material besteht. Dafür sprechen auch die unregelmäßig eingestreuten Holz-Stückchen. Besonders auffallend in den Pollendiagrammen dieser Schichtenfolge ist eine Abnahme der Pollendichte nach unten sowie ein regelmäßiges Auftreten von Hystrichosphaerideen.

$\mathrm{Da}$ die feinsandigen Schichten zwischen 5,93 $\mathrm{m}$ und $7,37 \mathrm{~m}$ vermutlich aus umgelagerten Schmelzwassersedimenten bestehen, ist die hier erbohrte Untere Mittelterrasse 2 vermutlich als zeitgleich bzw. etwas jünger als der saalezeitliche Inlandeisvorstoß an den Niederrhein anzusprechen.

Zwischen 5,27 $\mathrm{m}$ und 5,93 $\mathrm{m}$ schließt sich ein grobsandiger Mittelsand an, der von Fein- und MittelkiesLagen durchsetzt ist. Dieser Teil der Schichtenfolge trennt den oberen Teil der Bohrung Vorselaer bis $5,27 \mathrm{~m}$ von dem unteren Teil unter 5,93 $\mathrm{m}$ ab. Auch schwermineralanalytisch kommt dies durch die plötzliche Zunahme des Klinopyroxengehaltes und die Abnahme der stabilen Schwerminerale deutlich zum Ausdruck.
Von $4,77 \mathrm{~m}$ bis $5,27 \mathrm{~m}$ wurden die Vorselaer-Schichten durchbohrt. Es sind graue, glimmerführende Feinsande mit einem geringen Schluffanteil. Bei guter Pollenführung enthalten sie warmzeitliche Pollenspektren (siehe Abb. 5). Von Anfang an dominiert Alnus ganz eindeutig mit allerdings rückläufiger Tendenz von 52,2 auf 15,9\%. Der Eichenmischwald, fast ausschließlich durch Quercus vertreten, liegt zwischen 15,5 und $8,0 \%$. Etwas geringer ist der Anteil von Corylus. Mit Werten um $1 \%$ sind Carpinus und Taxus regelmäßig vorhanden. Abies dagegen fehlt. Läßt man Alnus außer Betracht, so beträgt der Anteil thermophiler Laubhölzer zwischen 15 und $25 \%$. Ferner kommen auch Hedera, Ilex und Acer vor sowie bei 5,1 m ein Pollenkorn von Fagus. Ab 4,9 m verändert sich die Vegetationszusammensetzung. Der NBP-Anteil steigt deutlich an, die NBP-Flora wird sippenreicher. Betula breitet sich kräftig aus und Empetrum erscheint wie auch Juniperus. Die Pollendichte nahm in den nachfolgenden Proben stark ab.

Es kamen weder präquartäre Sporomorphae noch Hystrichosphaerideen in diesem Profilabschnitt vor. Vergleicht man die vorliegenden Ergebnisse, die zweifellos warmzeitliche Pollenspektren nachgewiesen haben mit anderen Funden aus diesem Zeitabschnitt, so stimmen sie zwar mit den Untersuchungen nach MENKE (1968) aus der alten Grube in Wacken gut überein. Doch ist der in der Bohrung Vorselaer $\left(\mathrm{KB} 7^{\mathrm{B}} / \mathrm{C}\right)$ erfaßte Vegetationsabschnitt für eine gesicherte Parallelisierung zu kurz.

Das Pollenspektrum dieser Schichten kann eindeutig von den hangenden Eem-Torfen abgetrennt werden. Auffallend gegenüber den liegenden Schichten der Unteren Mittelterrasse 2 ist das völlige Fehlen von Hystrichosphaerideen und ein hoher Anteil präquartärer Pollenformen.

Die Vorselaer-Schichten zeigen weder sedimentologisch noch schwermineralanalytisch Hinweise auf eine Umlagerung. Trifft diese Annahme zu, hat man es bei den Vorselaer-Schichten mit einer Warmzeit innerhalb der späten Saale-Kaltzeit zu tun. Diese Einstufung ergibt sich aus der stratigraphischen Verknüpfung der Unteren Mittelterrasse 2 mit dem Inlandeisvorstoß. Die Vorselaer-Schichten müßten daher nach dem Inlandeisvorstoß und vor der Eem-Warmzeit entstanden sein. Diese stratigraphische Zuordnung legt eher eine Korrelation der Vorselaer-Schichten mit der Treene-Warmzeit nahe.

Sollten die Vorselaer-Schichten, entgegen allen Indizien, dennoch umgelagert sein, so kann es sich nur um eine Umlagerung über eine kurze Distanz gehandelt haben. Dies würde bedeuten, daß die VorselaerSchichten das Umlagerungsprodukt einer älteren Warmzeit darstellen. Die Umlagerung muß am Ende 


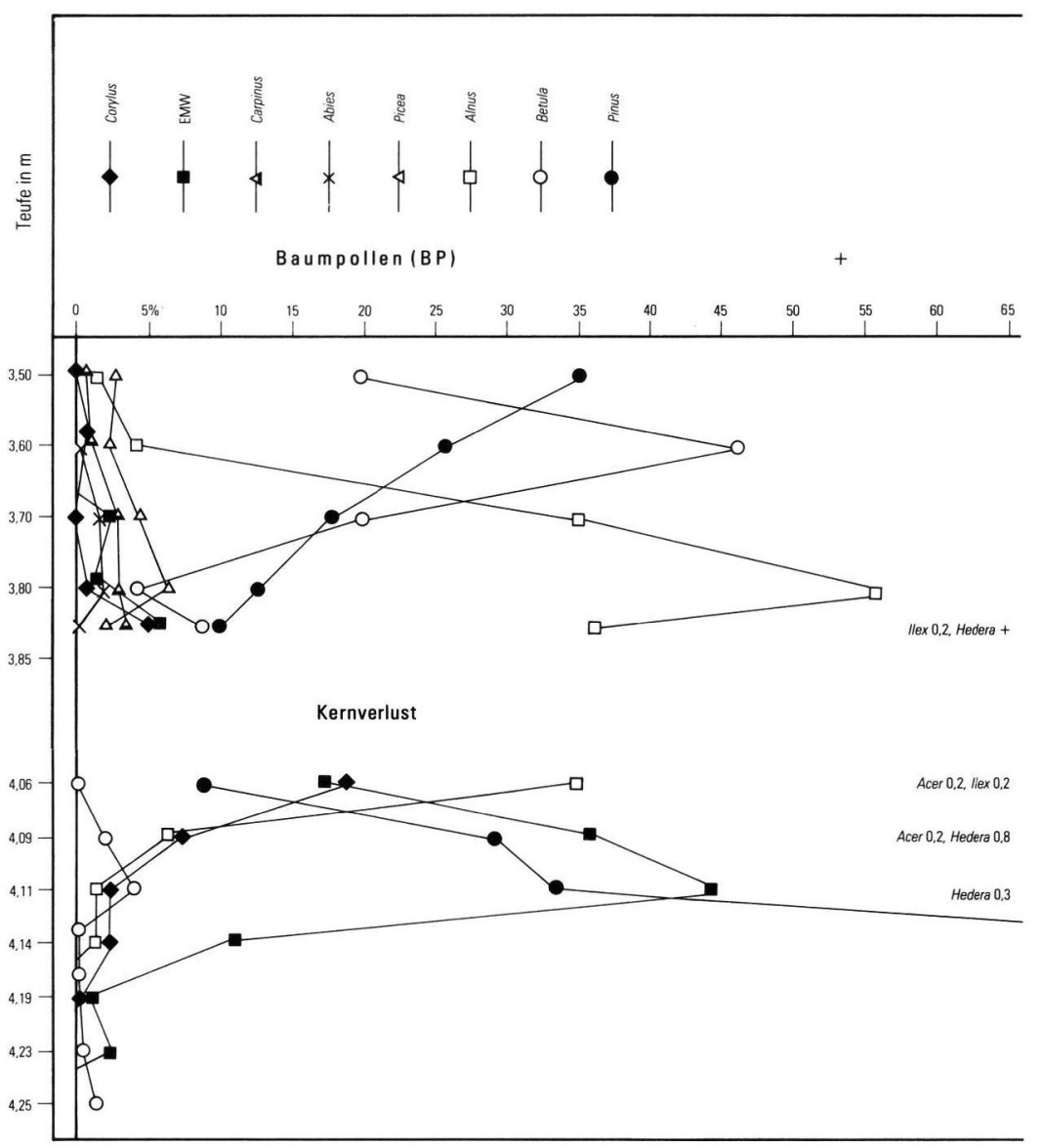

Abb. 5: Pollendiagramm der Bohrung Vorselaer $\left(\mathrm{KB} 7^{\mathrm{B}} / \mathrm{C}\right)$; vgl. Abb. 4.

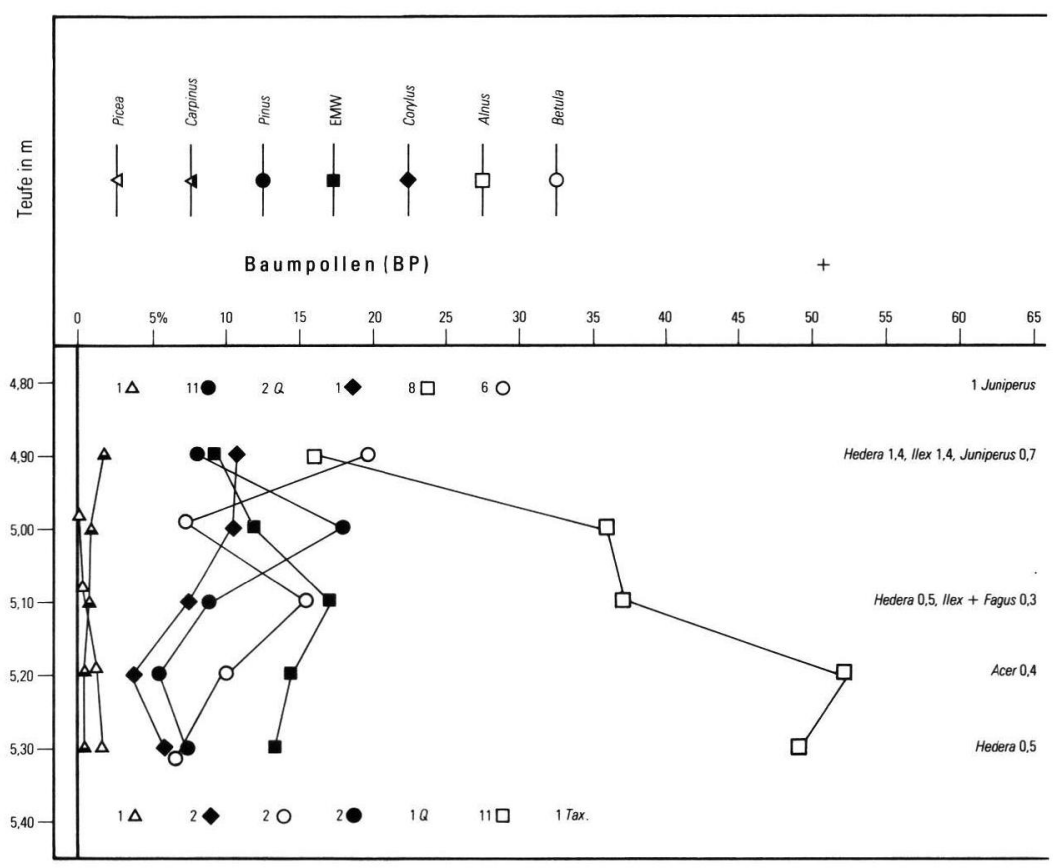



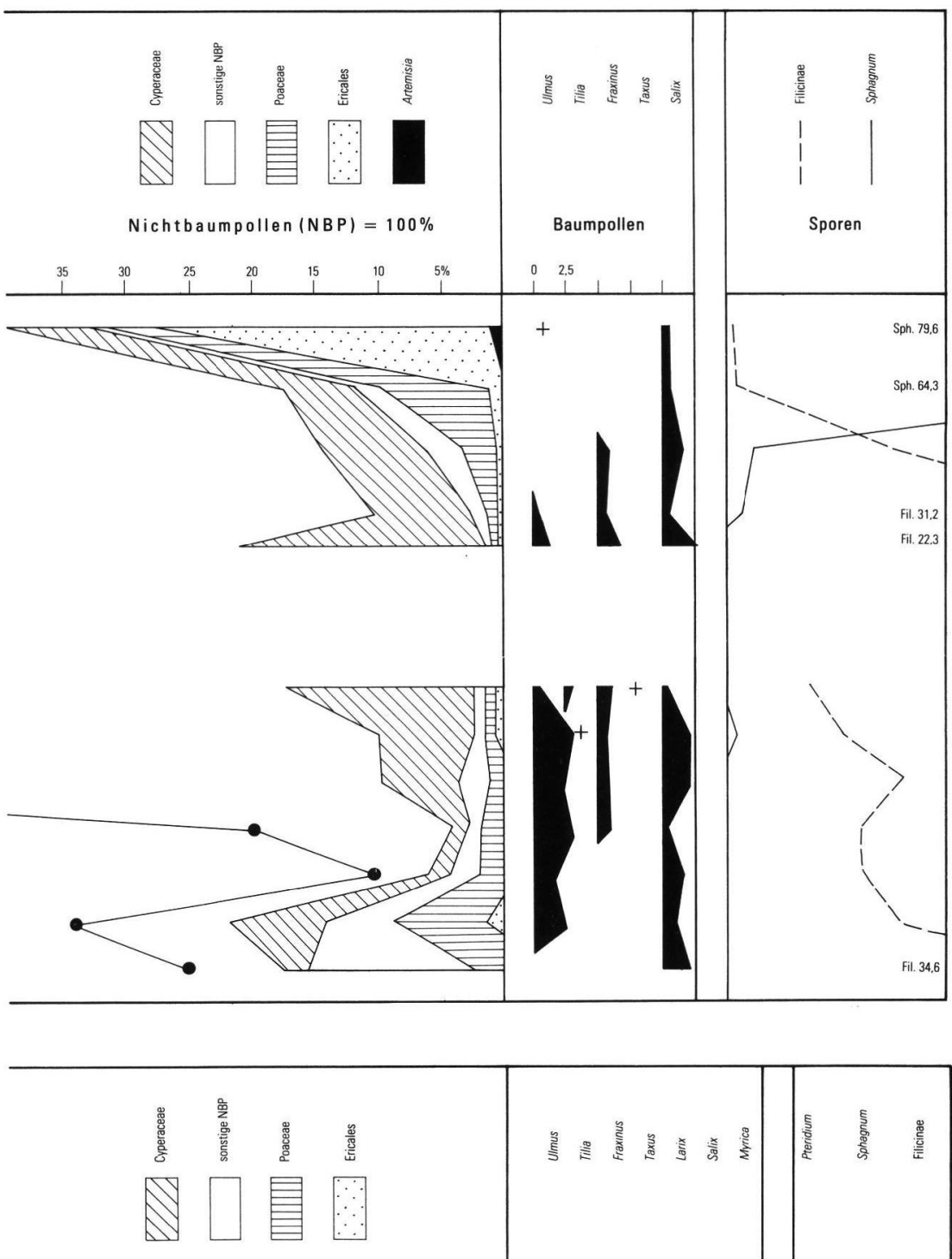

Nichtbaumpollen (NBP) $=100 \%$

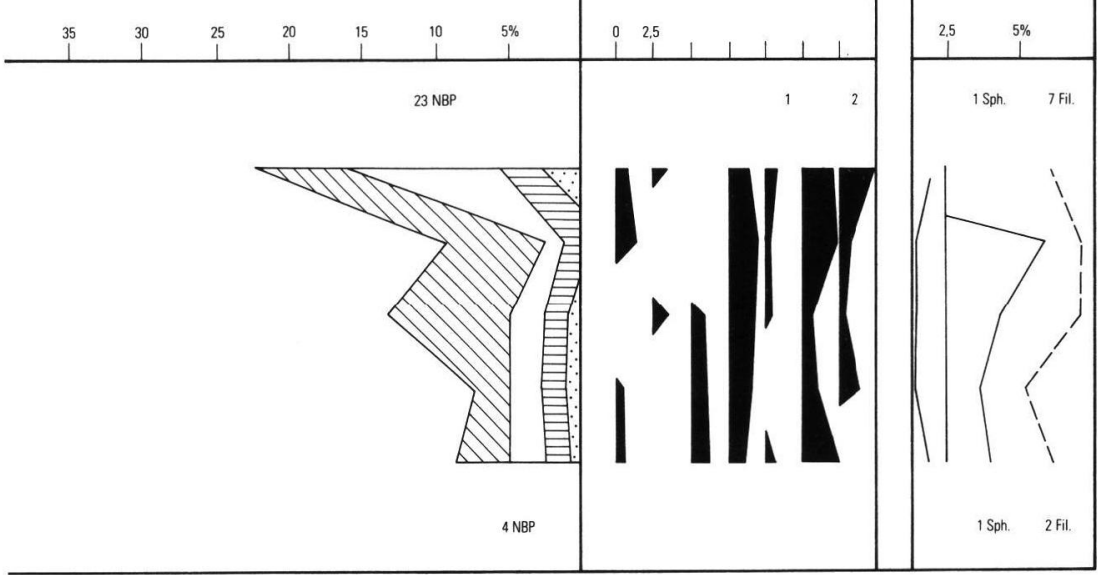


der Saale-Kaltzeit stattgefunden haben. In der Nähe der Bohrung Vorselaer müßten zu dieser Zeit Ablagerungen einer solchen Warmzeit angestanden haben. Eine Aufarbeitung von holsteinzeitlichen Sedimenten käme nur in Frage, wenn die Holstein-Schichten in unmittelbarer Nähe, etwa im gleichen Niveau, angestanden hätten wie die Vorselaer-Schichten. Da die Äquivalente der Holstein-Warmzeit in der Bohrung Vorselaer und der weiteren Umgebung, jedoch immer in Tiefen von mehr als $10 \mathrm{~m}$ vorkommen, könnte eine Aufarbeitung holsteinzeitlicher Ablagerungen nur dadurch erklärt werden, daß die Schichten der Holstein-Warmzeit in unmittelbarer Nähe von Vorselaer im Verband einer Stauchmoräne bis auf etwa $4 \mathrm{~m}$ unter Gelände aufgestaucht worden wären. Um eine Umlagerung holsteinzeitlicher Sedimente zu bewerkstelligen, müßte die gestauchte Zone südöstlich der Bohrung Vorselaer liegen, da eine Umlagerung nur von stromaufwärts gelegenen Gebieten her erfolgen kann. Für die Untere Mittelterrasse 2 ist dies der Fall, da sie zu einem erheblichen Teil aus umgelagerten Schmelzwasserablagerungen besteht, die während der Aufschotterung der Unteren Mittelterrasse 2 auch südöstlich Vorselaer angestanden haben. Für die Vorselaer-Schichten aber müßte für die Zeit ihrer Entstehung eine Stauchmoräne unmittelbar südöstlich Vorselaer gefordert werden. Nach den bisher vorliegenden Untersuchungen aber gibt es darauf keinerlei Hinweis. Anders in nordöstlicher Richtung. Dort konnte in wenigen Metern Tiefe, direkt unter den Ablagerungen der Niederterrasse, eine Stauchmoräne nachgewiesen werden.

Die Ergebnisse der Bohrung Vorselaer erlauben den vorsichtigen Schluß, daß es während der späten SaaleKaltzeit noch eine Warmzeit gegeben haben dürfte. Damit finden die Untersuchungen von LÜTTIG (1958), der erstmals eine Vollgliederung des Drenthe-Stadiums durchführte, am Niederrhein eine Bestätigung. Möglicherweise lassen sich die Vorselaer-Schichten mit dem Gerdau-Interstadial verknüpfen. Es bedarf jedoch noch weiterer Untersuchungen im Gebiet südöstlich von Vorselaer und Weeze, um die Stellung der Vorselaer-Schichten abzusichern. Sollte sich herausstellen, daß die Vorselaer-Schichten umgelagert sind, würde dies bedeuten, daß der saalezeitliche Inlandeisvorstoß mindestens $7 \mathrm{~km}$ weiter nach $\mathrm{W}$ gereicht haben muß als bisher angenommen. Dies würde wiederum bedeuten, daß der Rhein während des saalezeitlichen Inlandeisvorstoßes nur über den ViersenWalbecker Höhenrücken nach W hin abgeflossen sein kann. Auch für diese These gibt es bisher keine Hinweise.

Zwischen $4,25 \mathrm{~m}$ und $4,77 \mathrm{~m}$ wurden feinsandige Schluffe mit einem geringen Tonanteil erbohrt. Darin fanden sich unregelmäßig eingestreute Holzstücke.
Der NBP-Anteil in diesem Abschnitt der Schichtenfolge liegt deutlich über jenem der Vorselaer-Schichten. Die NBP-Flora wird sippenreicher. Ab 4,8 m ist die Tendenz zu einem ungünstiger werdenden Klima nicht zu übersehen.

Zwischen 3,60 m und 4,25 m wurden stark komprimierte organogene Ablagerungen der Eem-Warmzeit durchteuft. Da Kernverluste zu beklagen sind, können Schichtlücken nicht ausgeschlossen werden.

Das Pollendiagramm (vgl. Abb. 5) setzt mit der ausklingenden Zone II (nach MENKE \& TYNNI 1981) ein. Die Zonen III und IVa liegen vollständig vor und zeigen die bekannten Vegetationsabfolgen. Der Beginn der Zone IVb ist gerade noch angetroffen worden. Acer, Hedera und Ilex sowie in der Bohrung $\mathrm{KB} 7 \mathrm{C}$ Viscum wurden wiederholt nachgewiesen. Infolge Kernverlusts konnten die Beobachtungen erst gegen Ende der Hainbuchen-Fichten-Zeit (Zone V) wieder fortgeführt werden. Tilia und Taxus wurden nicht mehr angetroffen. Die weitere Vegetationsentwicklung bis in die Zone VII hinein ist durch lokale Vorgänge teilweise überdeckt. Zum Ende des Pollendiagramms breiten sich bei weiter ansteigender PinusKurve die Nichtbaumpollen, vor allem die Ericales, sowie Sphagnum kräftig aus.

Vollständiger wurde das eemzeitliche Torflager in Weeze erfaßt. Damals konnte durch VON DER BRELIE, MÜCKENHAUSEN \& REIN (1955) eine 2,5 m mächtige Torffolge bearbeitet werden, die auch die infolge Kernverlustes fehlenden Zonen enthielt. Allerdings überlagerte auch hier $A \ln u s$ wegen des Erlenbruchtorfes und die Cyperaceae wegen des Riedtorfes weite Abschnitte des Pollendiagramms.

Im Hangenden des Eem schließen sich unter einer ca. $1 \mathrm{~m}$ mächtigen Hochflutsanddecke Sande und Kiese der Älteren Niederterrasse an.

\section{Schriftenverzeichnis}

Braun, F. J. \& QuITzow, H. W. (1961): Die erdgeschichtliche Entwicklung der niederrheinischen Landschaft. Niederrh. Jb., 5: 11-21, 5 Abb., 1 Tab.; Krefeld.

Brelie, G. von der, Mückenhausen, A. \& Rein, U. (1955): Ein Torf aus dem Eiszeitalter im Untergrund von Weeze. - Niederrhein, 22: 1-4, 1 Abb.; Krefeld.

KempF, E. K. (1966): Das Holstein-Interglazial von Tönisberg im Rahmen des niederrheinischen Pleistozäns. Eiszeitalter u. Gegenwart, 17: 5-60, 7 Abb., 16 Tab.; Öhringen/Württ.

Klostermann, J. (1984), mit Beitr. von Nötting, J., PAas, W. \& ReHAGEN, H.-W.: Erläuterungen zu Blatt 4403 Geldern. - Geol. Kt. Nordrh.-Westf. 1 : 25000 , Erl., 4403 Geldern: 138 S., 8 Abb., 13 Tab., 3 Taf.; Krefeld (Geol. L.-Amt Nordrhein-Westfalen). 
KiostermanN, J. (1985): Versuch einer Neugliederung des späten Elster- und des Saale-Glazials der Niederrheinischen Bucht. - Geol. Jb., A 83: 3-42, 22 Abb., 1 Tab.; Hannover.

- (1986), mit Beitr. von NötTing, J., PAAs, W. \& ReHAGEN, H.-W.: Erläuterungen zu Blatt 4404 Issum. - Geol. Kt. Nordrh.-Westf. 1 : 25000, Erl. 4404 Issum: 120 S., 15 Abb., 9 Tab., 3 Taf.; Krefeld (Geol. L.-Amt Nordrhein-Westfalen).

LÜTTIG, G. (1958): Heisterbergphase und Vollgliederung des Drenthe-Stadiums. - Geol. Jb., 75: 419-430, 6 Abb., 1 Tab.; Hannover.

MenKE, B. (1968): Beiträge zur Biostratigraphie des Mittelpleistozäns in Norddeutschland (pollenanalytische Untersuchungen aus Westholstein). - Meyniana, 18: 35-42, 6 Abb.; Kiel.
Menke, B. \& Tynni, R. (1984): Das Eeminterglazial und das Weichselfrühglazial von Rederstall / Dithmarschen und ihre Bedeutung für die mitteleuropäische Jungpleistozän-Gliederung. - Geol. Jb., A 76: 3-120, 18 Abb., 7 Tab., 9 Taf.; Hannover.

MÜLLER, H. (1974): Pollenanalytische Untersuchungen und Jahresschichtenzählungen an der eemzeitlichen Kieselgur von Bispingen/Luhe. - Geol. Jb., A 21: 149-169, 5 Abb., 3 Tab.; Hannover.

ZAGWIJN, W. H. (1973): Pollenanalytic studies of Holsteinian and Saalian Beds in the Northern Netherlands. - Meded. Rijks Geol. Dienst, N.S., 24: 139-156, 12 Abb.; Haarlem.

Manuskript eingegangen am 3.7.1987,

Nachträge Nov. 1987. 